\title{
Correction to: Analysis of Microbial Diversity and Community Structure of Peanut Pod and Its Surrounding Soil in Peanut Rot Epidemic Area
}

\author{
Meijing $\mathrm{He}^{1,2} \cdot$ Weiming Sun ${ }^{3} \cdot$ Shunli Cui ${ }^{4} \cdot$ Guojun $\mathrm{Mu}^{4} \cdot$ Lifeng Liu $^{4} \cdot$ Wei Guo ${ }^{5}$
}

Published online: 14 August 2021

(c) Springer Science+Business Media, LLC, part of Springer Nature 2021

\section{Correction to: Current Microbiology (2021) 78:2173-2182 https://doi.org/10.1007/s00284-021-02471-3}

The original version of this article unfortunately contained a mistake. The acknowledgements and conflict of interest section were missing from this article and which are given below.

Acknowledgements This work was financially sponsored by National Natural Science Foundation of China (31801394 and 31771833) and the China Agriculture Research System (CARS-13) and Postdoctoral Science Foundation of Hebei Province (B2017005028). The authors sincerely thank the Xuan Chen Biological Technology Co., Ltd. (Shaanxi, China) for help with data analysis.

\section{Declarations}

Conflict of interest The authors declare no conflict of interest.

The original article can be found online at https://doi.org/10.1007/ s00284-021-02471-3.

Lifeng Liu

liulifeng@hebau.edu.cn

Wei Guo

1787421502@qq.co

1 College of Plant Protection, Hebei Agricultural University, Baoding, China

2 College of Agronomy and Forestry, Hebei North University, Zhangjiakou, China

3 College of Agronomy and Biotechology, Hebei Normal University of Science \& Technology, Qinhuangdao, China

4 College of Agronomy, Hebei Agricultural University, Baoding, China

5 Graduate School, Chinese Academy of Agricultural Sciences, Beijing, China
Publisher's Note Springer Nature remains neutral with regard to jurisdictional claims in published maps and institutional affiliations. 